\title{
COMPORTAMENTO REOLÓGICO DE ARGAMASSA DE ASSENTAMENTO E REVESTIMENTO COM ADIÇÃO DE REJEITO DE CONSTRUÇÃO CIVIL E CINZAS VOLANTES
}

\author{
D.N.P.CARDOSO ${ }^{1 *}$, K.F.CASTRO ${ }^{1}$, J.A.S. SOUZA ${ }^{1}$, C.A.G. SOUZA ${ }^{1}$, \\ A.M.P.F. FELIPE ${ }^{1}$ \\ ${ }^{1}$ PPEQ-Programa de Pós-Graduação em Engenharia Química \\ Laboratório de Reologia - Instituto de Tecnologia - Universidade Federal do Pará \\ *e-mail: dnpcardoso@gmail.com.br
}

RESUMO - As argamassas podem ser consideradas como um conjunto de partículas sólidas em suspensão (agregado miúdo), aglomerantes e água (pasta de cimento). O trabalho teve como objetivo a verificação do comportamento reológico de quatro traços de argamassas, em diferentes proporções de cimento Portland CP II Z 32, rejeito da construção civil (RCC) e cinza volante (CV). O RCC foi beneficiado e caracterizado pelas análises de espectrofotometria e difração de raios-X. Para o ensaio reológico utilizou-se o viscosímetro com sensor tipo cilindros coaxiais $\mathrm{SV}_{1}$ em taxas de 0 e $600 \mathrm{~s}^{-1}$ no intervalo de tempo de $120 \mathrm{~s}$. Os resultados mostraram que a incorporação de cinza volante é o principal fator para a mudança do comportamento de reópetico para tixotrópico, nas argamassas. Os dados experimentais das formulações propostas ajustaramse ao modelo reológico de Herschel-Bulkley.

\section{INTRODUÇÃO}

A argamassa pode se apresentar como uma mistura homogênea complexa de materiais orgânicos (polímeros) ou inorgânicos (agregado miúdo) e produtos especiais incorporados, chamados de aglomerantes (cimento Portland), que se tornam ativos na presença de água, além de materiais especiais chamados de aditivos, que podem variar as suas características físicas e químicas de acordo com a sua utilização. A mistura destes constituintes em proporções estudadas deve ser homogênea de acordo com o tipo de argamassa pretendida (SABBATINI 1984).

O estudo das propriedades reológicas das argamassas no estado de pré cura é de fundamental importância, tanto na sua aplicabilidade quanto no seu processamento (RAMACHANDRAN, 2001)

Fatores diversos influenciam na reologia das argamassas, dentre esses fatores destacam-se, as características das composições da mistura (teor de água, tipo de agregado, cimento e adição de aditivos) e as condições experimentais que foram realizadas as misturas. O tamanho e a geometria das partículas que constituem o produto têm a capacidade de influenciar na redução da aglomeração, atuando na diminuição da viscosidade aparente, facilitando o seu escoamento, já a 
quantidade de água tem por finalidade promover as reações de hidratação e facilitar as ligações das partículas de cimento e dos agregados (SENFF, 2010 e GASTALDINI, 1999).

\subsection{Objetivo}

Este trabalho tem como objetivo a avaliação das características reológicas de quatro formulações de argamassas dentro de um intervalo de tempo de 120s, determinado pelos efeitos iniciais de cura do material, em função da incorporação de rejeito da construção civil (RCC) como carga e cinza volante $(\mathrm{CV})$ como aglomerante, em substituição parcial do cimento.

\section{MATERIAIS E MÉTODOS}

O material coletado (RCC) passou por processos de beneficiamento de acordo com a norma ABNT NBR 7217. Todo o material separado (alvenaria e reboco) foi britado, moído e classificado em peneiras a fim da amostra obedecer aos requisitos da norma ABNT NBR 7211, em seguida uma amostra do material foi submetida a analise de espectrometria de fluorescência de raios $-X$ e difração de raios $-X$.

A cinza volante usada foi proveniente da refinaria de alumina Norsk Hydro SA, localizada no município de Barcarena-Pa. A sua produção ocorre em caldeiras de leito fluidizado circulante a partir da combustão do carvão mineral, foram realizadas analises granulométricas nas CV para a verificação se há necessidade prévia de tratamento para ser utilizada como pozolona. O cimento Portland utilizado foi classificado como tipo (CP II - E 32), a principal escolha desse material foi por ser um cimento com poucas adições de pozolanas. A água utilizada foi proveniente do sistema de abastecimento da Universidade Federal do Pará.

\subsection{PREPARO DAS AMOSTRAS}

Foi elaborada a relação água aglomerante (a/ag) de 0,35 sendo que este valor foi definido a partir de estudos de literatura, sendo adaptado de modo experimental de acordo com o tempo de trabalho, determinado pelos efeitos de cura do material, em que $35 \%$ de $\mathrm{H}_{2} \mathrm{O}$ refere-se ao valor total de $100 \%$ de mistura ( RCC, cimento e CV). Sendo 65\% de sólidos da mistura como mostra a Tabela 1

Tabela 1 - Proporção dos materiais usados nos traços

\begin{tabular}{ccccc}
\hline \multicolumn{5}{c}{ MATRIZ DE ANÁLISE } \\
Traços & RCC (\%) & Cimento (\%) & CV (\%) & Água (\%) \\
\hline $\operatorname{Tr} 1$ & 85 & 5 & 10 & 35 \\
$\operatorname{Tr} 2$ & 80 & 5 & 15 & 35 \\
$\operatorname{Tr} 3$ & 75 & 5 & 20 & 35 \\
$\operatorname{Tr} 4$ & 70 & 5 & 25 & 35 \\
\hline
\end{tabular}

\subsection{PARÂMETROS DAS ANALISES REOLÓGICOS}

Para a obtenção das curvas de fluxo utilizou-se taxas de 0 a $600 \mathrm{~s}^{-1}$, no tempo de $120 \mathrm{seg}$, em temperatura de $28^{\circ} \mathrm{C}$. 
A programação para das analises de curva de histerese foi elaborada segundo a metodologia de (ARAÚJO, 2009) adaptada.

(I) Rampa de aumento de taxa de cisalhamento $(\gamma)$ de 0 a $600 \mathrm{~s}^{-1}$ em $120 \mathrm{~s}$;

(II) Permanência a $600 \mathrm{~s}^{-1}$ por $30 \mathrm{~s}$;

(III) Rampa de diminuição de taxa de cisalhamento $(\gamma)$ de 600 a $0 \mathrm{~s}^{-1}$ em $120 \mathrm{~s}$.

\section{RESULTADOS E DISCUSSÃO CARACTERIZAÇÃO DAS MATÉRIAS PRIMAS}

Os resultados obtidos na análise granulométrica do RCC, após a cominuição mostram que esses valores encontram-se na faixa de $4,8 \mathrm{~mm}$ e $0,075 \mathrm{~mm}$ o que permite enquadrar como um agregado miúdo, como mostra a Figura 1 abaixo.

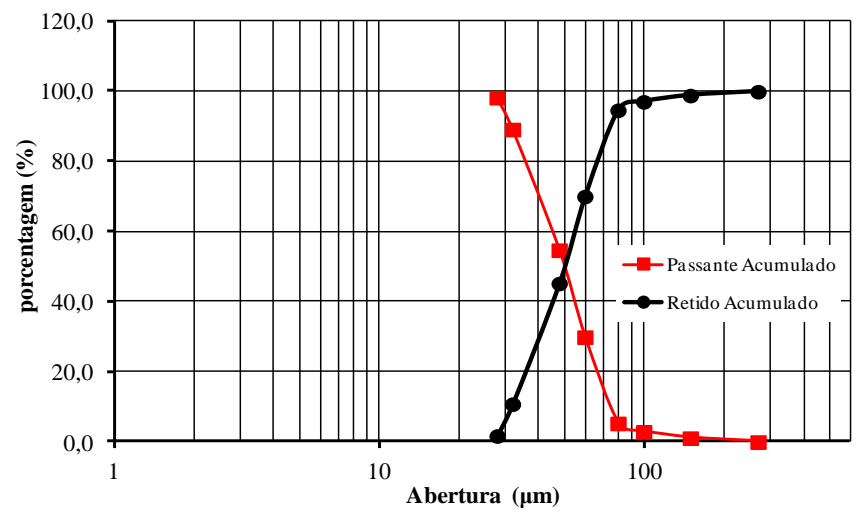

Figura 1 - Análise granulométrica.

Espectrofotometria de fluorescência de raios-X

A Tabela 2 mostra que o resíduo de construção civil é composto em sua maioria por óxidos de sílica, em sua minoria óxidos de alumínio, ferro e potássio o que caracteriza um agregado miúdo para produção de argamassa.

Tabela 2 - Fluorescência de raios-X.

\begin{tabular}{cc}
\hline $\begin{array}{l}\text { REJEITO DE CONSTRUÇÃ̃O CIVIL } \\
\text { Componente }\end{array}$ & $\begin{array}{c}\text { Conc. }(\%) \\
\mathrm{SiO}_{2}\end{array}$ \\
$\mathrm{Al}_{2} \mathrm{O}_{3}$ & 93,41 \\
$\mathrm{Fe}_{2} \mathrm{O}_{3}$ & 0,59 \\
$\mathrm{~K}_{2} \mathrm{O}$ & 0,32 \\
Perda ao fogo & 0,19 \\
\end{tabular}

Difração de raios $-\mathrm{X}$

A Figura 2 representa a caracterização da amostra por difração de raios $-X$. De acordo com as intensidades das raias de difração, verifica-se que, dos compostos cristalinos, o quartzo é mais 
frequente, o que caracteriza o rejeito de construção civil como um dos materiais constituintes da argamassa.

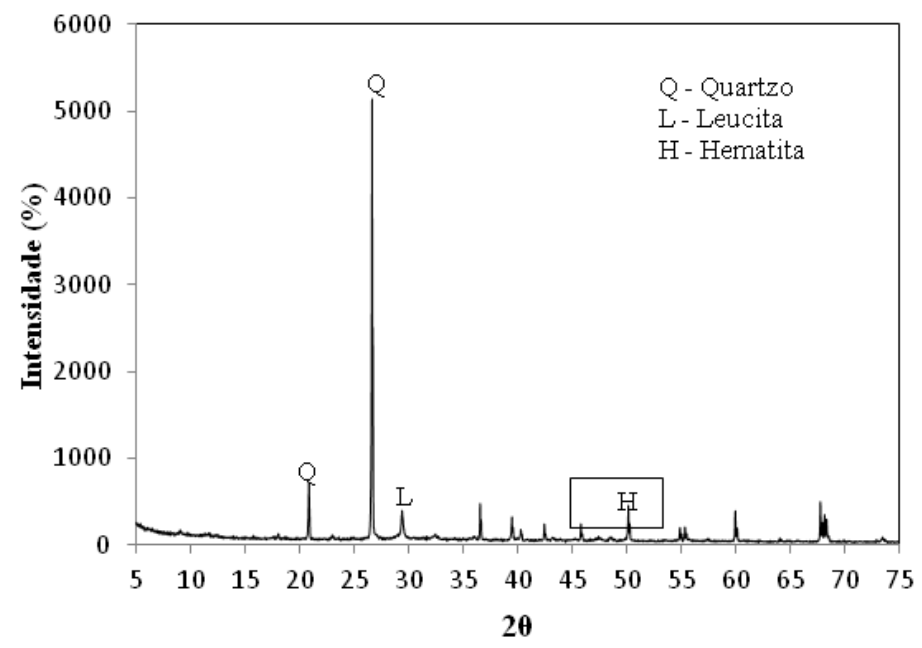

Figura 2- Difração de raios $-\mathrm{X}$ do resíduo de construção civil.

\section{RESULTADOS DAS ANALISES REOLÓGICAS}

$\mathrm{Na}$ Figura 3, Observou-se que os traços possuem em comum o comportamento de fluidos não-newtonianos dependentes do tempo. Estudos de literatura mostram que sistemas constituído por partículas sólidas em suspensão, dependendo do teor de material incorporado apresentam características de fluidos não-newtonianos dependentes do tempo, podendo apresentar características Reópeticas ou Tixotrópicas (SANTOS, 1989), apresentando a diminuição da viscosidade aparente conforme o aumento da taxa de cisalhamento (SCHARAMM, 2006).

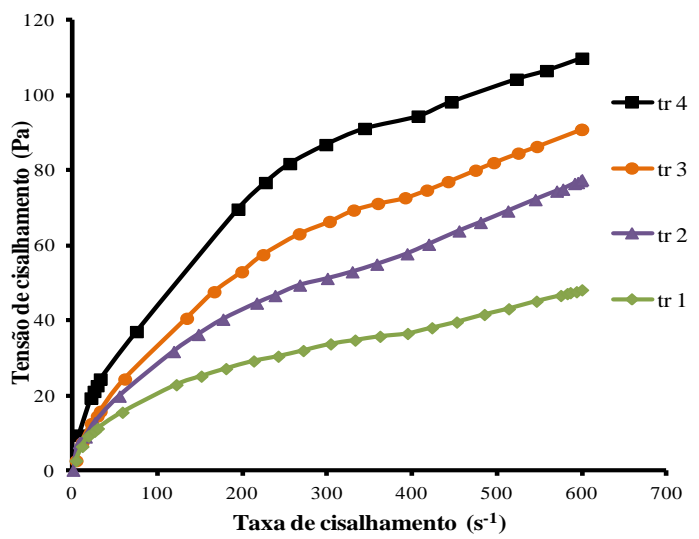

Figura 3 - Curva de fluxo (tensão versus taxa) para cada traço 
Observa-se na Figura 4, que as curvas reológicas do traço 1 e demais traços possuem menores valores de viscosidade aparente em relação ao traço 4 . A justificativa para essa mudança gradual é que a contribuição do RCC (70\%) em conjunto com a CV (25\%) e o teor de água incorporado nos traços, contribuiu para o aumento da viscosidade aparente.

Em relação ao RCC, que está em concentração maior que a $\mathrm{CV}$, suas partículas finas e assimétricas quando se encontram hidratadas, fazem surgir forças de atração entre si favorecendo a formação de aglomerados que aumentam a viscosidade aparente da suspensão (OLIVEIRA, 2000)

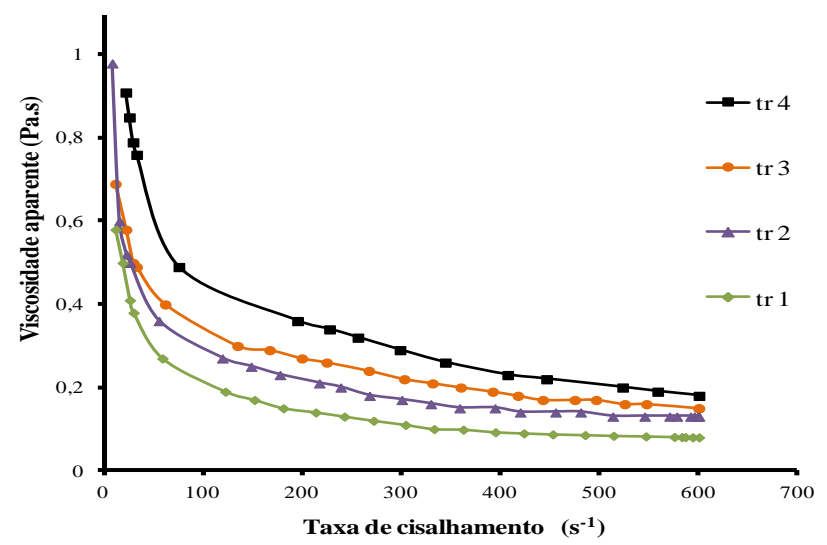

Figura 4- Curva de fluxo (viscosidade versus taxa) para cada traço

Na Figura 5 Verifica-se a mudança gradual das características apresentadas no traço 1 (reopético), em comparação aos traços 2 e 3 (tixotrópicos ). Isto pode ser atribuído a composição do traço 1, por ser constituída de partículas finas, assimétricas e inertes (RCC) pois essas partículas apresentam pouca atividade pozolânica em relação a quantidade de cimento Portland (CP) e isenta de CV. Partículas finas e assimétricas quando hidratadas fazem surgir forças de atração entre si favorecendo a formação de aglomerados que aumentam a viscosidade aparente da suspensão, originando o comportamento reopético (OLIVEIRA, 2000).
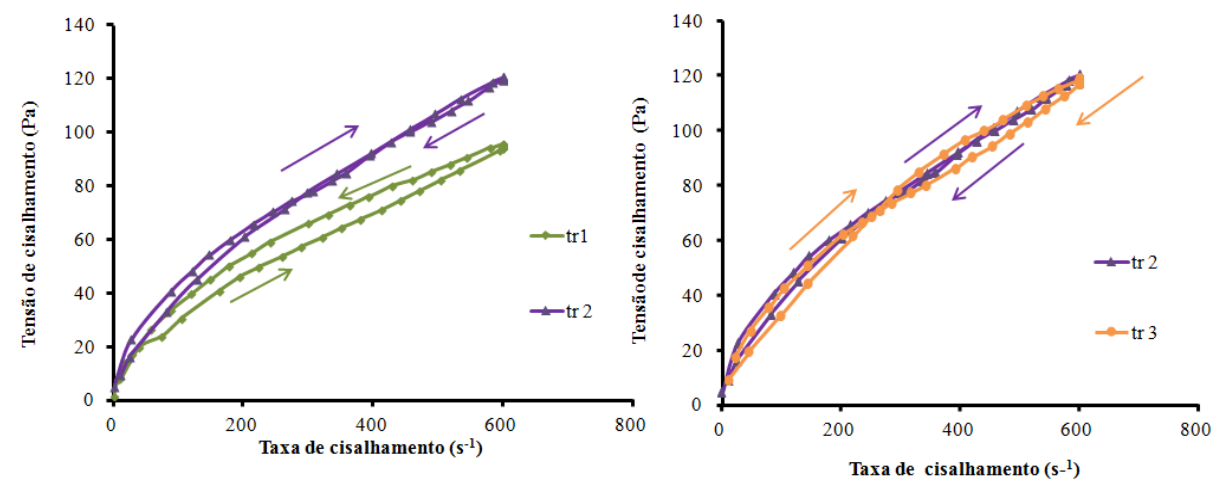

Figura 5 - Histerese reológica para os traços 1 e 2 em comparação com os traços 2 e 3 


\section{LCoBEe}

19 a 22 de outubro de 2014

Florianópolis/SC

Comparando as curvas dos traços 3 e 4 da Figura 6, verifica-se que essa mudança já está consolidada no traço 4 (tixotrópica), devido a composição desse traço ter sido influenciado pelos efeitos filer e pozolânico provenientes da CV. A melhoria do comportamento induzida pela presença de $\mathrm{CV}$, referente ao efeito pozolanico é devida a elevada quantidade de água adsorvida pelo carbono presente nas $\mathrm{CV}$, pois quanto maior for o índice de desestruturação do material mais energia será requerida para quebrar um maior número de ligações entre as partículas de ligante, que são responsáveis pela sua aglomeração. Ao contrario quanto menor for o valor deste índice mais defloculada encontra-se a pasta e mais reversível é o seu comportamento, que neste caso ocorreu de uma forma gradual de acordo com a incorporação da CV e diminuição do RCC.

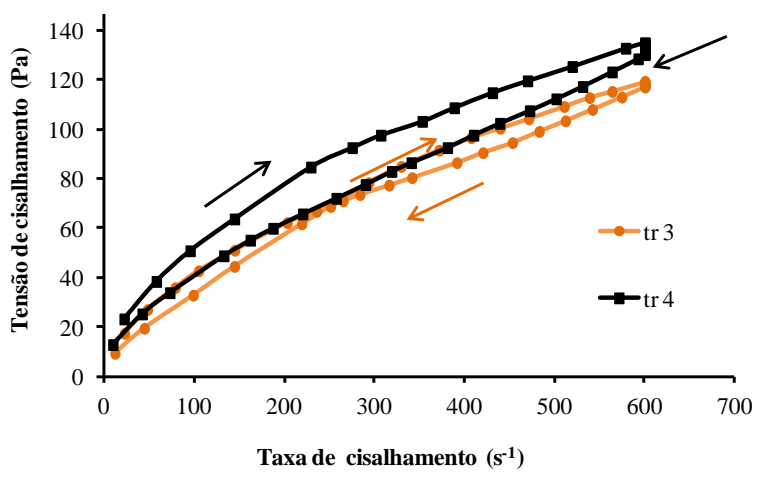

Figura 6 - Histerese reológica para os traços 3 e 4.

A Tabela 3 apresenta os ajustes propostos para os resultados experimentais nos traços 1 a 4 aos modelos reológicos de Bingham e Herschel-Bulkley. Os resultados mostraram melhores correlações com o modelo reológico de Herschel-Bulkley, de acordo com o tempo de ensaio, evidentes nos traços 2, 3 e 4 A expressão proposta por Herschel-Bulkley é dependente do parâmetro $\left(\tau_{0}\right)$, índice de consistência $(\mathrm{k})$ e o índice de comportamento do fluido(n), fatores esses que envolvem propriedades que podem levar em consideração os efeitos das reações que ocorrem durante o processo, o que fazem do modelo de Herschel-Bulkley, o mais significativo em termos experimentais (CAMÕES, 2002)

Tabela 3: Modelos reológicos de Bingham e Herschel-Bulkley para os traços 1 a 4

\begin{tabular}{|c|c|c|c|c|c|c|c|c|}
\hline \multirow{2}{*}{ ARGAMASSA } & \multirow{2}{*}{ MÉDIA } & \multicolumn{3}{|c|}{ Bingham } & \multicolumn{4}{|c|}{ Herschel-Bulkley } \\
\hline & & $\tau_{0}(\mathrm{~Pa})$ & $\overline{\eta_{p}(\mathrm{~Pa} . \mathrm{s})}$ & $\mathrm{R}^{2}$ & $\tau_{0}(\mathrm{~Pa})$ & $\mathrm{K}$ & $\mathrm{n}$ & $\mathrm{R}^{2}$ \\
\hline \multirow{4}{*}{$\operatorname{tr} 1$} & & 15,64 & 0,1317 & 0,9921 & 4 & 1,041 & 0,6912 & 0,9986 \\
\hline & & 13,95 & 0,05872 & 0,975 & 4,565 & 2,623 & 0,4502 & 0,9969 \\
\hline & & 19,57 & 0,1639 & 0,9942 & 7,592 & 0,985 & 0,7314 & 0,999 \\
\hline & MÉDIA & 16,387 & 0,118 & 0,987 & 5,386 & 1,550 & 0,624 & 0,998 \\
\hline \multirow{4}{*}{$\operatorname{tr} 2$} & & 16,16 & 0,1342 & 0,992 & 2,619 & 1,246 & 0,6685 & 0,9993 \\
\hline & & 17,42 & 0,1037 & 0,9803 & 6,221 & 1,567 & 0,6482 & 0,9969 \\
\hline & & 22,26 & 0,1511 & 0,9908 & 4,655 & 1,969 & 0,6183 & 0,9998 \\
\hline & MÉDIA & 18,613 & $\mathbf{0 , 1 3 0}$ & 0,988 & 4,498 & 1,594 & 0,645 & 0,999 \\
\hline \multirow{4}{*}{$\operatorname{tr} 3$} & & 22,12 & 0,1746 & 0,9917 & 9,361 & 2,085 & 0,6349 & 0,9998 \\
\hline & & 21,61 & 0,127 & 0,9683 & 9,184 & 7,444 & 0,4147 & 0,997 \\
\hline & & 27,04 & 0,1827 & 0,9811 & 6,416 & 5,052 & 0,5187 & 0,9976 \\
\hline & MÉDIA & 23,59 & 0,161 & 0,980 & 8,320 & 4,860 & 0,523 & 0,998 \\
\hline \multirow{4}{*}{$\operatorname{tr} 4$} & & 24,96 & 0,1413 & 0,9722 & 21,76 & 8,419 & 0,414 & 0,9979 \\
\hline & & 33,49 & 0,1467 & 0,9427 & 22,52 & 22,81 & 0,2945 & 0,995 \\
\hline & & 33,39 & 0,1878 & 0,9743 & 23,02 & 5,801 & 0,4977 & 0,9992 \\
\hline & MÉDIA & 30,613 & 0,159 & 0,963 & 22,433 & 12,343 & 0,402 & 0,997 \\
\hline
\end{tabular}




\section{CONCLUSÕES}

A influência das propriedades das CV em conjunto com RCC mostraram que ocorreu uma mudança significativa nas características das argamassas devido a elevada absorção de água com formação de uma cadeia cristalina de produtos hidratados, tendo como consequência a redução de água no sistema resultando no aumento da tensão de cisalhamento e da viscosidade aparente.

A influência das propriedades das CV em conjunto com RCC de acordo com os reogramas mostraram que ocorreu uma mudança gradativa do comportamento reópetico para tixotrópico, ao longo dos traços analisados, o que evidência os efeitos filer e pozolânico nos traços avaliados, sendo mais acentuado a partir dos traços 2, 3 e 4, devido o aumento do teor de CV,o que ocasionou a diminuição das áreas das histereses ao longo do tempo em que ocorreu o ensaio, permitindo confirmar a destruição irreversível da estrutura da argamassa.

O modelo de Bingham apresentou correlações que podem ser considerar satisfatória, porém os resultados mostram que, os dados experimentais ajustaram-se melhor ao modelo de HerschelBulkley. O estudo realizado foi bastante promissor, pois permitiu avaliar elementos construtivos de baixo custo, acelerando o processo de gerenciamento de resíduos sólidos, o que ocasiona a uma diminuição das áreas utilizadas para destinação desses rejeitos.

\section{NOMENCLATURA}

- CV - cinza volante

- RCC- rejeito da construção civil

- Tr- traços elaborados para as analises das argamassas

- a/ag - relação água aglomerante

- $\tau_{0}$ - tensão limite de escoamento

- $\eta_{\mathrm{p}}$ - viscosidade aparente

- $\mathrm{k}$ - índice de consistência

\section{REFERÊNCIAS}

ARAÚJO, M. S.; FRANÇA, S. C. A.; BARBATO C. N.; Estudo Reológico de polpa de Bauxita da Região Nordeste do Pará. In: JORNADA DE INICIAÇÃO CIENTIFICA DO CETEM/MCT, 17. Anais... 2009.p.22-28. Rio de Janeiro.

GASTALDINI, A. L. G. \& ISAIA, G. C. Chloride permeability of high performance concrete with mineral addition: binary and ternary mixtures. INTERNATIONAL CONFERENCE ON HIGH PERFORMANCE CONCRETE, 2. Gramado, Brasil, Proceedings, 1999. 1 CD-ROM. 
OLIVEIRA, I., STUDART, A., PILlEGI, R., PANDOLFELli, V. Dispersão e em pacotamentode partículas - Princípios e aplicações em processamento cerâmico. $18^{a}$ ed. São Paulo, Fazendo Arte, 2000.

RAMACHANDRAN, V.S., BEAUDOIN, J.J. Handbook of analytical techniques in concrete science and technology, New York, Noyes / William Andrew, 2001

SABBATINI, F. H. O processo construtivo de edifícios de alvenaria estrutural sílicocalcária. São Paulo. 1984. 298 p. Dissertação (Mestrado) - Escola Politécnica da Universidade de São Paulo.

SANTOS, P. S. Ciência e Tecnologia de Argilas. 2.ed. rev. e ampl. São Paulo: Edgar Blucher, 1989.

SCHRAMM, G; Reologia e reometria: fundamentos teóricos e práticos. Tradução de Cheila G. Mothé; Denise Z. Correia; Hans M. Petri; Michelle Gonçalves; Tatiana Carestiato. São Paulo: Artliber Editora, 2006.

SENFF, L.; HOTZA, D; REPETTE, W.L.; Comportamento reológico de pastas de cimento com adição de sílica ativa, nanossílica e dispersante policarboxílico Revista Matéria, v. 15, n. 1, pp. $012-020,2010$

CAMÕES, A, Betões de Elevado Desempenho com Incorporação de Cinzas Volantes, Tese de Doutoramento, Universidade do Minho, Guimarães, Portugal (2002). 